\title{
Power Estimation in Mobile Communication Systems
}

\author{
Lenin Gopal, Ashutosh Kumar Singh \& Veeramani Shanmugam \\ School of Engineering and Science \\ Curtin University of Technology \\ CDT 250, Miri, Sarawak, Malaysia
}

Tel: 60-85-443-932Ｅ-mail: lenin@curtin.edu.my

\begin{abstract}
Accurate power estimation has an important role for power control and handoff decisions in mobile communications. Window based weighed sample average power estimators are commonly used due to their simplicity. In practice, the performances of these estimators degrade severely when the estimators are used in the presence of correlated samples. In this paper performances of the three local mean power estimators namely, sample average, optimum unbiased and maximum likelihood estimators, are analysed in the presence of correlated samples. The variance of the estimators is used as performance measures. Finally, the simulation results show that the performances of the optimum unbiased and maximum likelihood estimators are very good as compared to the performance of the sample average estimator.
\end{abstract}

Keywords: Power estimation, Local mean power, Rayleigh fading, Mobile communications

\section{Introduction}

In mobile communication systems, local mean power estimation is very important and it must be an accurate estimate of the received signal power. Some system function like channel access, handoff, and power control use local mean signal level to indicate the wireless communication link quality.

Mockford, Turkmani and Parsons (1990) analysed local mean signal variability in rural areas. The local mean of the signal envelope was described by a lognormal distribution. The power spectral density of the local mean signal was estimated along rural routes. Goldsmith, Greenstein and Foschini (1994) studied the error statistics of real time power measurements in cellular channels with multipath and shadowing. The authors considered two measurement methods (filtering the squared envelop, and filtering the logarithm of the squared envelop) and two filter types (integrate-and-dump and RC). Accurate measurements were obtained by filtering either the logarithm of the detected power or the power itself. Valenzuela, Landon and Jacobs (1997) explored techniques for the measurement of local mean signal strength at 900 $\mathrm{MHz}$ and $2 \mathrm{GHz}$. Linear averaging technique was used to estimate the local mean and ray tracing propagation model was used to evaluate different methods of calculating the local mean signal strength for indoor environment. Manohar Das and Cooprider (1997) introduced new techniques for detection of changes in the local mean of a signal. The first one utilized a discrete cosine transform (DCT) based data compression principle, and the second one was based on a robust piecewise linear approximation (PLA) of the given signal. The results of experimental studies were compared with the performance of the two methods with the existing filtered derivative method. Wong and Cox (1999) derived the optimal local mean signal level estimator for the Rayleigh fading environment and compared with the sample average estimator. Variance of the two estimators were estimated and compared with the Cramer-Rao Lower bound. The $5^{\text {th }}$ and $95^{\text {th }}$ percentiles of the estimators were obtained by computer simulation. Antilog Rayleigh (ALR) distribution was used to estimate the signal variation in a Rayleigh fading environment.

Young-Chai Ko and Mohamed-Slim Alouini (2001) presented two local mean power estimation techniques over Nakagami fading channels. Chai Ko and Mohamed-Slim Alouini presented maximum likelihood as well as minimum variance unbiased estimators for the local mean signal power estimation. De Jong and Herben (2001) presented new method for the computation of local mean power from individual multipath signals predicted by two-dimensional ray tracing based on an expression for the spatial average of the received power, which takes into account the spatial correlation between signals. The presented method is based on the spatial average of the received power over each pixel area. Avidor and Mukerjee (2001) investigated the possibility of obtaining better estimates or prediction of the path loss between a mobile and the surrounding base station by processing more measurements, including older measurements of received power that are not used by current deployed algorithm. The current or near future value of the local mean 
received power including the shadow loss was estimated by using the algorithm. Tepedelenlioglu, Sidiropoulos and Giannakis (2001) derived the maximum likelihood and median filtering for power estimation in mobile communication system. Linear filtering techniques for power estimation was compared with the maximum likelihood (ML) estimator and the median filtering techniques for power estimation was compared with the linear filtering, maximum likelihood (ML) estimator and uniformly minimum variance unbiased (UMUV) estimator. Jiang, Sidiropoulos and Giannakis (2003) proposed a scalar Kalman-Filter-based approach for improved local mean power estimation. The performance of the Kalman filter (KF) was compared with the window based estimators, like the sample average estimator of (Goldsmith, Greenstein \& Foschini, 1994) the uniformly minimum variance unbiased (UMVU) estimator of (Wong \& Cox, 1999) and the maximum likelihood (ML) estimator of (Tepedelenlioglu, Sidiropoulos and Giannakis, 2001).

In this paper, the performances of the sample average estimator, the optimum unbiased estimator and maximum likelihood estimator are studied with the use of uncorrelated and correlated samples (Rayleigh fading signal). For correlated samples, the variances of the three estimators are obtained while varying the correlation between the samples.

The first part of this paper introduces the system model for the local mean power estimation using uncorrelated and correlated samples. Following that, the three estimators details are included. The performances of the three local mean power estimators and conclusion are included in the subsequent sections.

\section{System Model}

\subsection{Uncorrelated Fading Samples}

The model for the uncorrelated fading samples at the log amplifier output of the receiver is shown in Fig .1. The uncorrelated samples can be expressed as

$$
Y=r_{i}^{2}+r_{q}^{2}
$$

The output of the amplifier is expressed as

$$
X=20 \log Y
$$

$X$ has a probability density function called the Antilog Rayleigh (ALR). The ALR probability density function is given by

$$
f_{x}(X)=(\ln 10) \frac{10^{\frac{x}{10}}}{20 p} \exp \left(-\frac{10^{\frac{x}{10}}}{2 p}\right)
$$

where $p=E\left(Y^{2}\right) / 2$ is the power of each phase component.

\subsection{Correlated Fading Samples}

Fig. 2 shows the simulator model for correlated samples (Rayleigh fading signal). A block of $N_{f}=1024$ uncorrelated samples are generated and the maximum Doppler frequency shift $f_{m}=50 \mathrm{~Hz}$ is specified to produce proper Doppler shift. The (Smith, 1975) steps are used to generate series of simulated Rayleigh fading signal with proper Doppler spread and correlation between the samples. The correlated output samples are fed into the lograthemic amplifier. The output of the amplifier can be expressed as in eq.2.

\section{Local Mean Power Estimators}

In this section, the conventional local mean power estimators for the Rayleigh fading channel are briefly reviewed.

\subsection{Sample Average Estimator}

Due to the rapid changing characteristics of the Rayleigh fading process, an estimate of local mean power is obtained by averaging the samples $X$. The average of the received samples as follows,

$$
E_{S A}=\frac{1}{N} \sum_{j=1}^{N} X_{j}
$$

where $N$ is the window size, $X_{j}$ is the received power measurement.

\subsection{Optimum Unbiased Estimator}

The optimum unbiased estimator was derived in (Wong, 1999) as follows.

$$
E_{O U}=10\left[\log T-\frac{H_{N-1}}{\ln 10}\right]
$$

Where

$$
T=\sum_{j=1}^{N} 10^{X_{j} / 10}
$$




$$
\text { and } \quad H_{N}=1+\frac{1}{2}+\frac{1}{3}+\frac{1}{4}+\ldots \ldots \ldots \ldots \ldots+\frac{1}{N}
$$

for $N=1,2,3, \ldots H_{N}$ is known as the $N^{\text {th }}$ harmonic number.

\subsection{Maximum Likelihood Estimator}

In (Tepedelenlioglu, 2001), the maximum likelihood estimator for local mean power estimation was derived as follows.

$$
E_{M L}=10[\log (T)-\log (N)]
$$

where

$$
T=\sum_{j=1}^{N} 10^{X_{j} / 10}
$$

\section{Simulation Results and Comparisons}

In this section, the performances of the three estimators, sample average estimator, optimum unbiased estimator and maximum likelihood estimator are compared. Simulations are carried out in uncorreleated and correlated samples environment. The channel parameters are obtained by the multipath fading generator as in Fig. 1 and 2 .

\subsection{Effect of Number of Samples, $N$}

Fig. 3 and 4 show the performance of the three estimators for uncorrelated and correlated samples. All the three estimators' variance gradually decreases for increasing number of samples. The variance obtained with optimum unbiased estimator and maximum likelihood estimators are consistently lower than that obtained with sample average estimator. From Fig. 3, the sample average estimator requires 50 samples to achieve $0.65 \mathrm{~dB}^{2}$ variance, but optimum unbiased and maximum likelihood estimators need 30 samples to achieve the $0.65 \mathrm{~dB}^{2}$ variance for uncorrelated samples.

Fig.4 shows the variance of the three estimators for correlated samples. From the Fig.4, the sample average estimator requires 200 samples to achieve $0.65 \mathrm{~dB}^{2}$ variance but the optimum unbiased and maximum likelihood estimators require only 100 samples to achieve the same performance. Optimum unbiased and maximum likelihood estimators consistently perform better than the sample average estimator over the entire range of number of samples tested for correlated samples.

Fig. 5 and 6 compare the performance of the $5^{\text {th }}$ and $95^{\text {th }}$ percentiles of the three estimator output for uncorrelated and correlated samples.In Fig.5, 50 samples are needed for the difference between the two percentile lines to be $2 \mathrm{~dB}$ for optimum unbiased and maximum likelihood estimators. About 85 samples are needed for sample average estimator to obtain the $2 \mathrm{~dB}$ differences between the two percentile lines. In Fig. 6 approximately 150 samples are required to achieve the $2 \mathrm{~dB}$ differences between the two percentile lines for optimum unbiased and maximum likelihood estimators. About 400 samples are required to achieve the similar performance with the sample average estimator for correlated samples.

\subsection{Effect of Doppler Frequency, $f_{m}$}

Fig. 7 and 8 show the relation between the variance of the estimators with normalised Doppler frequency. Doppler frequency of the Rayleigh fading signal is varied from $15 \mathrm{~Hz}$ to $300 \mathrm{~Hz}$. $M=2$ sample distances is used in the simulation. Sample average estimator has $17.6 \mathrm{~dB}^{2}$ variance at low normalised frequency $(0.01)$. The performance of the sample average estimator is improved at high Doppler frequency. The variance of the optimum unbiased and maximum likelihood estimators is $13 \mathrm{~dB}^{2}$ at low normalised frequency (0.01). The optimum unbiased and maximum likelihood estimators have better performance as compared to the sample average estimator for the tested range of Doppler frequency.

In Fig.8, the variance of the three estimators is compared over various normalized Doppler frequencies. Sample distance used in the simulation is $M=20$ and the Doppler frequency is varied from $15 \mathrm{~Hz}$ to $300 \mathrm{~Hz}$. Variance of the sample average estimator is $3.2 \mathrm{~dB}^{2}$ at 0.01 normalized Doppler frequency. Sample average estimator achieves the same performance at 0.08 normalized Doppler frequency for 2 sample distance. Optimum unbiased and maximum likelihood estimators require 20 sample distance to obtain $2.4 \mathrm{~dB}^{2}$ variance for 0.01 normalized Doppler frequency. Both estimators achieve the similar performance for 2 sample distance at 0.09 normalized Doppler frequency. The optimum unbiased and maximum likelihood estimators have very low variance as compared to the sample average estimator for 20 sample distance. The variance of the estimators is very high at low Doppler frequency and decreases at high Doppler frequency.

\subsection{Effect of Correlation Between Samples}

Fig.9 shows the relation between the variance of the estimators with the autocorrelation between the samples. 2 sample distances is used in the simulation. Optimum unbiased and maximum likelihood estimators have very low variance as compared to sample average estimators. The variance of all the estimators is very high at 0.9 to 1 auto-covariance. The variances of the estimators reduce for decreasing value of the autocorrelation between the samples.

In Fig.10, the performance of the three estimators is compared with the autocorrelation between the samples. 20 sample distance is used in the simulation and the Doppler frequency is varied from $15 \mathrm{~Hz}$ to $300 \mathrm{~Hz}$. The variance of the 
estimators is reduced by six times at 0.9 to 1 autocorrelation of the samples as compared to 2 sample distance simulation. Optimum unbiased and maximum likelihood estimators have very low variance as compared to the sample average estimators for the tested range of the autocorrelation of the samples. Performance of the estimators improves for increasing value of the sample distance used in the simulation. The variances of the three estimators reduce for increasing value of the sample distance.

\section{Conclusions}

In this paper, the performance of the local mean power estimators is analysed for uncorrelated and correlated samples (Rayleigh fading channel). The comparisons are made between the sample average estimator with optimum unbiased and maximum likelihood estimators. Optimum unbiased and maximum likelihood estimator requires much less samples to achieve the desired result as compared to the sample average estimator using uncorrelated samples. All the three estimators require more test samples to achieve the desired result using correlated samples as compared to the performance of the estimators using uncorrelated samples. The performances of the three estimators improve for the increased number of tested samples.

Finally, for the correlated samples, the required sample distance is investigated to achieve a similar performance of the uncorrelated samples. Large numbers of samples distances are required for the correlated samples to achieve similar performance of the uncorrelated samples. It is also noted that the higher the normalized Doppler frequency, the better is the estimator performance. Similarly, the higher the correlation between two samples, the poorer is the estimator performance.

\section{References}

Tao Jiang., Sidiropoulos, N. D., \& Giannakis B. Giannakis. (2003). Kalman Filtering for Power Estimation in Mobile Communications. IEEE Trans. On Wireless Communications, Vol.2, No-1, pp.151-161.

Avidor, D., \& Mukherjee, S. (2001). Estimation and Prediction of the Local Mean Signal Power in Mobile System. Veh. Technol. Conference, VTC 2001 Spring. IEEE VTS $53^{\text {rd }}$, Vol.4, 6-9 pp.2751-2755.

De Jong, Y. L. C., \& Herben, M. H. A. J. (2001). Prediction of Local Mean Power using 2-D Ray-Tracing-Based Propagation Models. IEE Trans.on. Vehicular Tech._Vol.50, No.1, pp.325-337.

Tepedelenlioglu,.C., Sidiropoulos, N.D., Giannakis B. Giannakis. (2001). Median Filtering for Power Estimation in Mobile Communication systems. in Proc. 3rd IEEE Signal Processing workshop on Signal Processing Advances in Wireless Communications, Taiwan, pp.229-231.

Young Chai Ko., \& Mohamed Slim Alouini. (2001). Estimation of the Local Mean Power over Nakagami Fading Channels. $12^{\text {th }}$ IEEE International Symposium on personal, Indoor and Mobile Radio Communications, Vol.1, pp c-107c-112.

Wong, D., \& Cox, D. C. (1999). Estimating Local Mean Signal Power Level in a Rayleigh Fading Environment. IEEE Trans. on Veh. Technol., Vol-48, No-3, pp.956-959.

Manohar Das., \& Cooprider, A. (1997). New Technologies for Detection of Changes in the Local Mean of a Signal. in proc.of the $40^{\text {th }}$ Midwest Symbosium on circuit and systems, Vol.2, 3-6, pp.849-852.

Valenzuela, R. A., Landon, O., \& Jacobs, D. L. (1997). Estimating Local Mean Signal Strength of Indoor Multipath Propagation. IEEE Trans. On Veh. Technol., Vol.46, No 1.

Goldsmith, A. J., Greenstein, L. J., \& Foschini, G. J. (1994). Error Statistics of Real-Time Power Measurements in Cellular Channels with Multipath and Shadowing. IEEE Trans on Veh.Technol, Vol.43, No.3, pp.439-446.

Mockford, S., Turkmani, A. M. D., \& Parsons, J. D. (1990). Local Mean Signal Variability in Rural Areas at 900 MHz. in Proc. $40^{\text {th }}$ vehicular Technology Conf.. pp. 610-615.

Smith, J. I. (1975). A Computer Generated Multipath Fading Simulation for Mobile Radio. IEEE Transactions on Vehicular Technology, Vol. VT-24, No.3, pp. 39-40. 


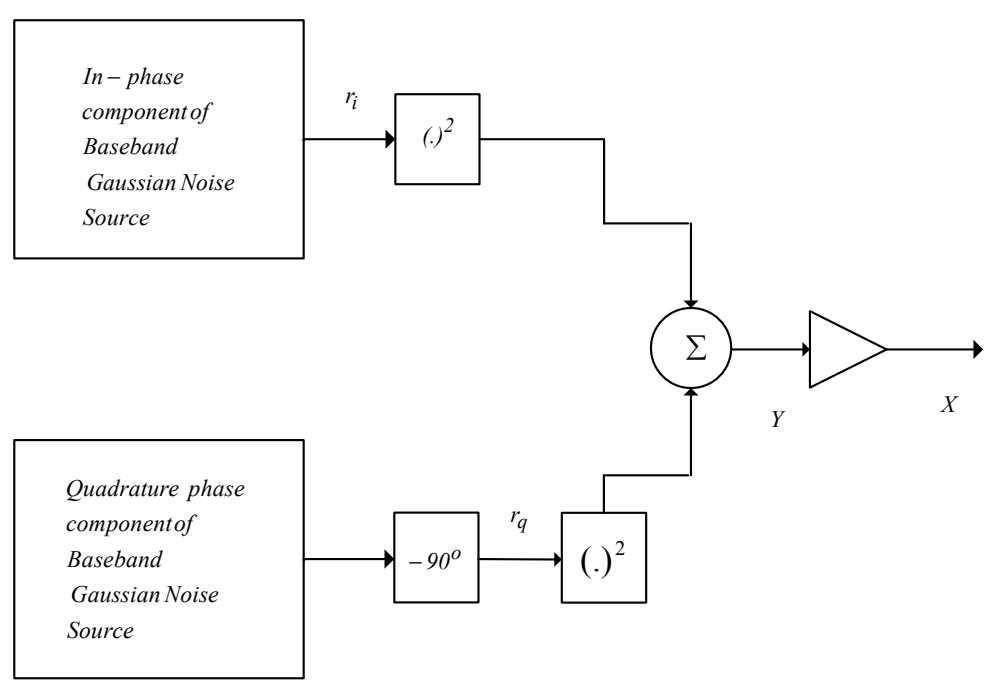

Figure 1. Simulator of uncorrelated fading samples.

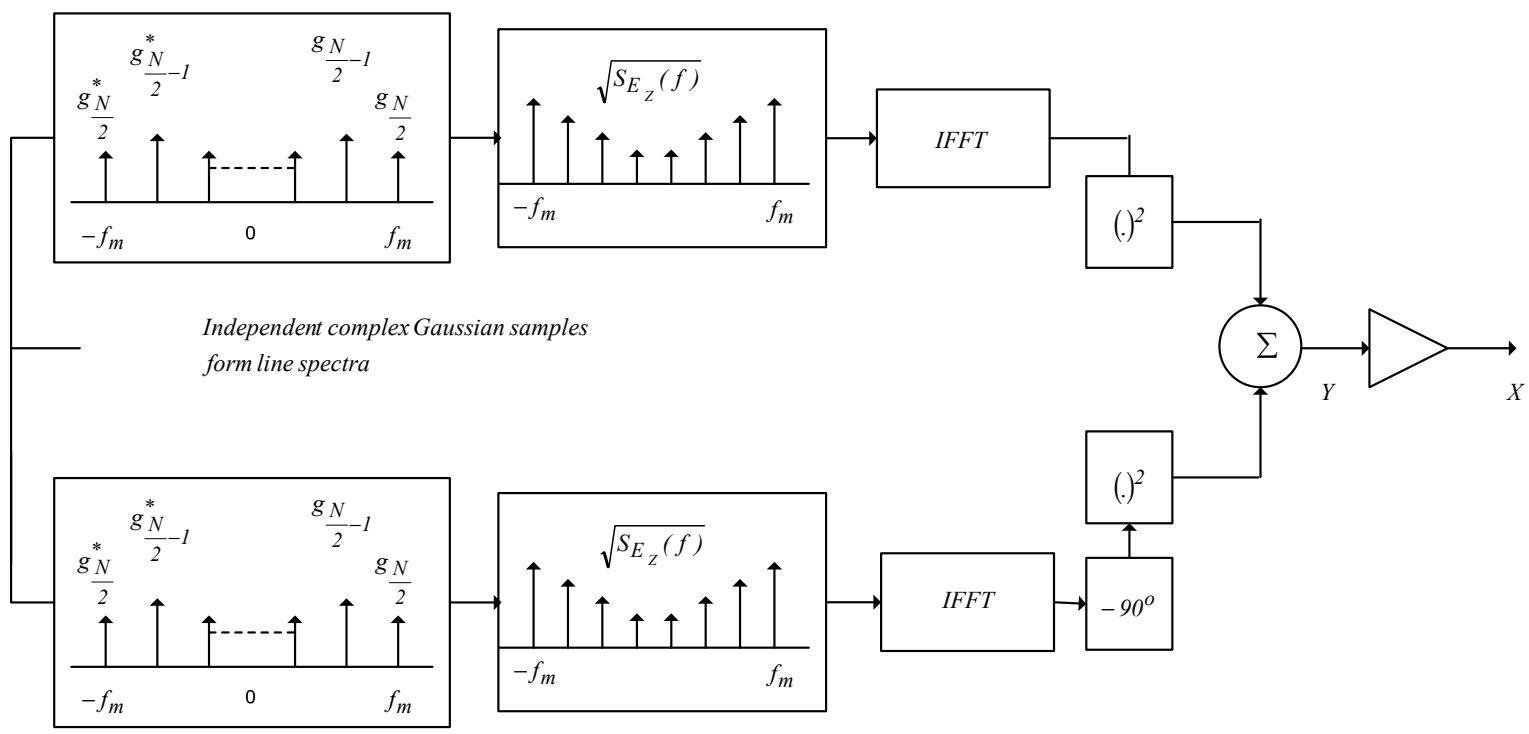

Figure 2. Simulator of correlated fading samples. 


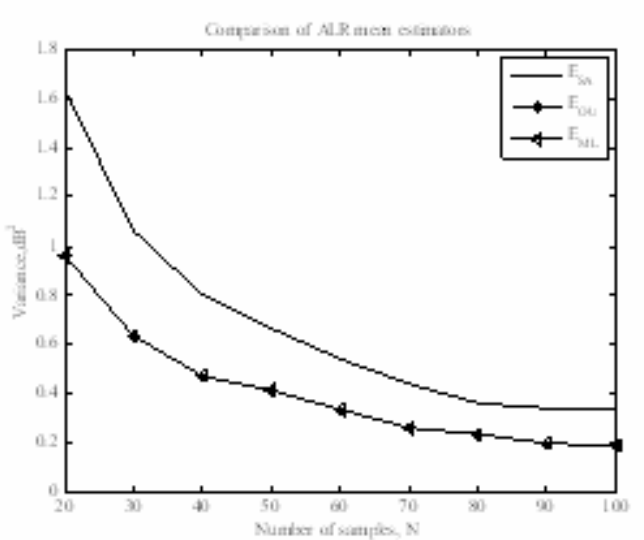

Fig. 3. Variance of the estimators for uncorrelated s

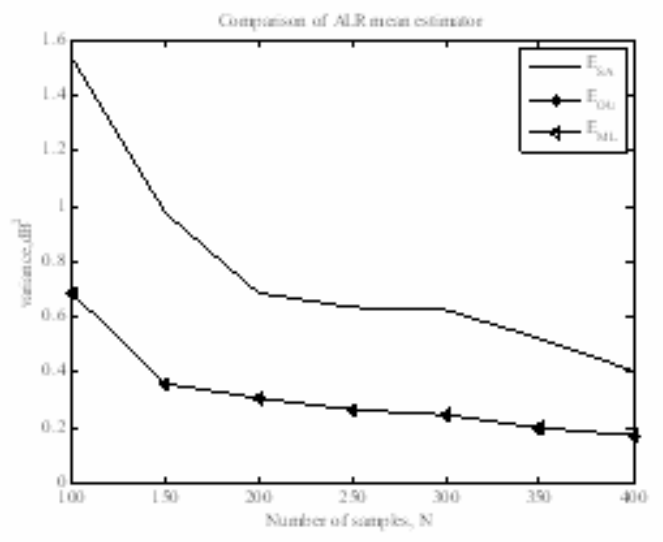

Fig. 4. Variance of the estimators for correlated samples.

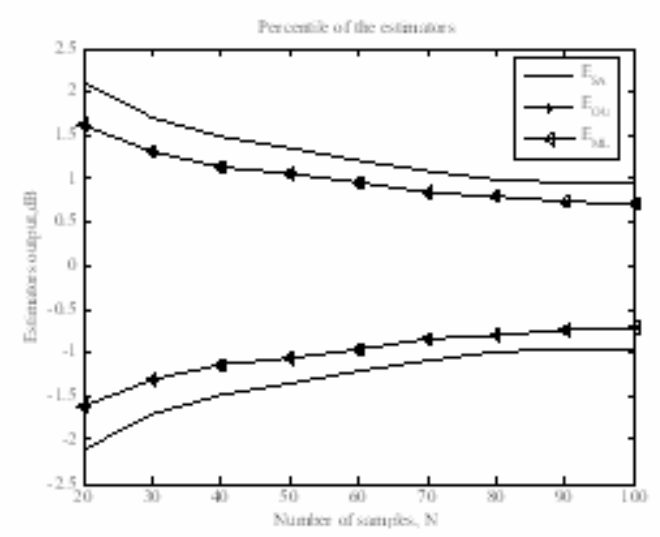

Fig. 5. The $5^{\text {th }}$ and $95^{\text {th }}$ percentiles of the estimat.

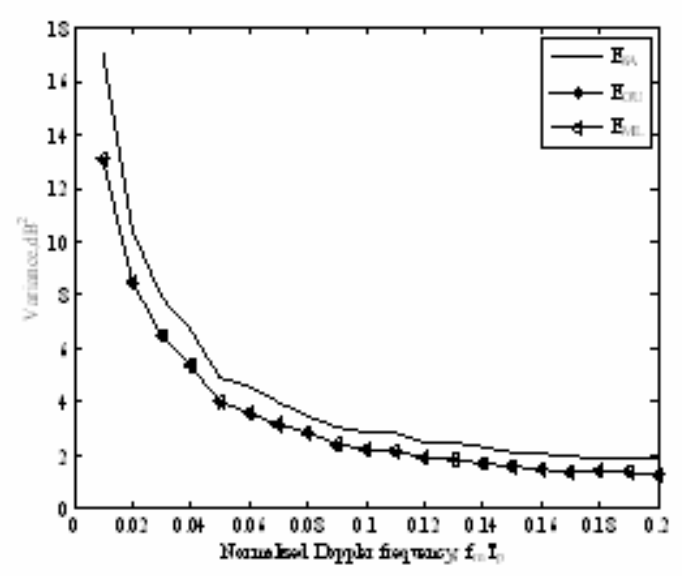

Fig. 7. Variance of the three estimators $\left[f_{m}=15 \mathrm{~Hz}\right.$, $30 \mathrm{~Hz} \ldots 300 \mathrm{~Hz}$, Number of samples per second $N N_{f}=1 / T_{p}=1500, N=20, M=21$

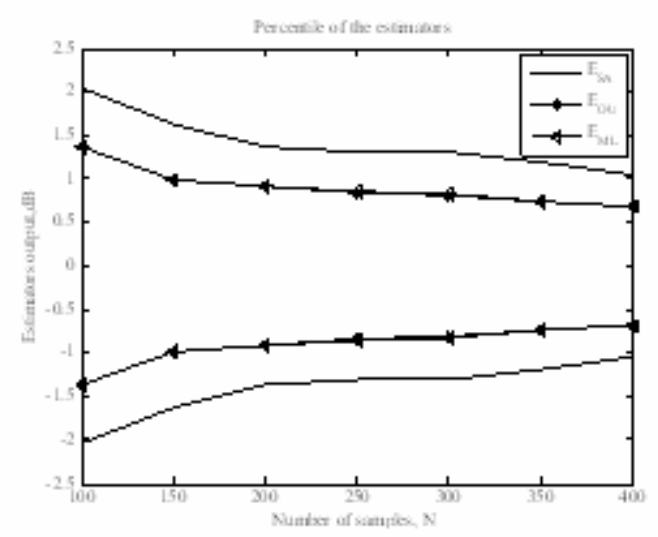

Fig. 6. The $5^{\text {th }}$ and $95^{\text {th }}$ percentiles of the estimators

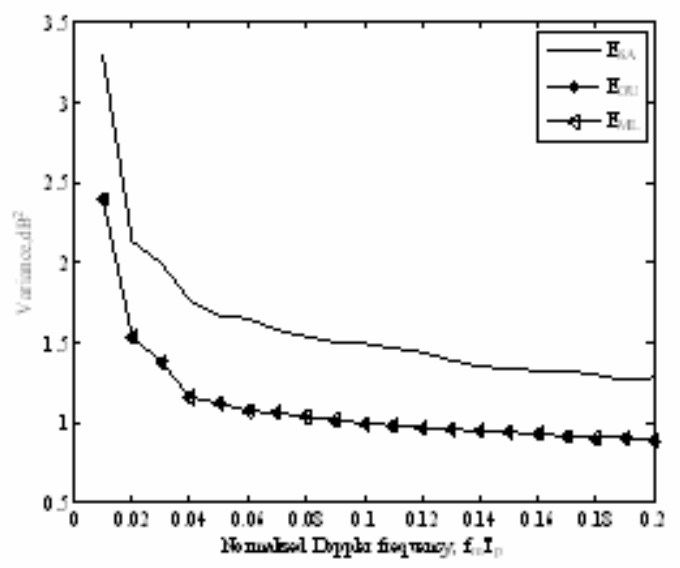

Fig. 8. Variance of the three estimators $\left[f_{m}=15 \mathrm{~Hz}, 30 \mathrm{~Hz}\right.$, .., $300 \mathrm{~Hz}$, Number of samples per second $N_{f}=1 / T_{p}=1500$, $N=20, M=201$ 


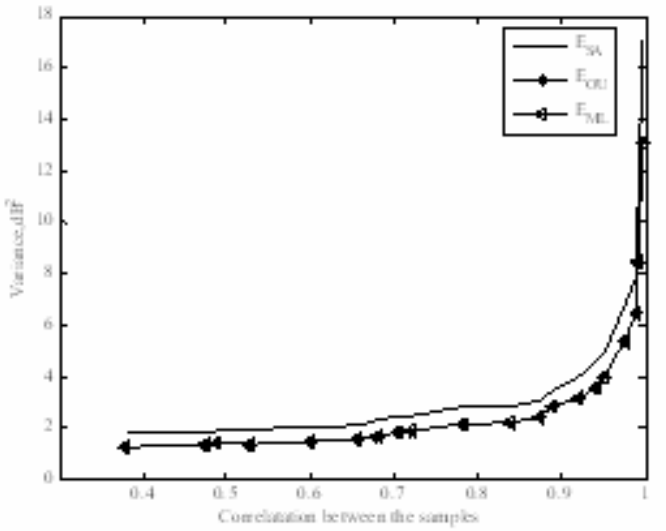

Fig. 9. Variance of the three estimators $\left[f_{m}=15 \mathrm{~Hz}\right.$, $30 \mathrm{~Hz} \ldots 300 \mathrm{~Hz}$, Number of samples per second $\left.N=1 / T_{p}=1500, N=20, M=2\right]$

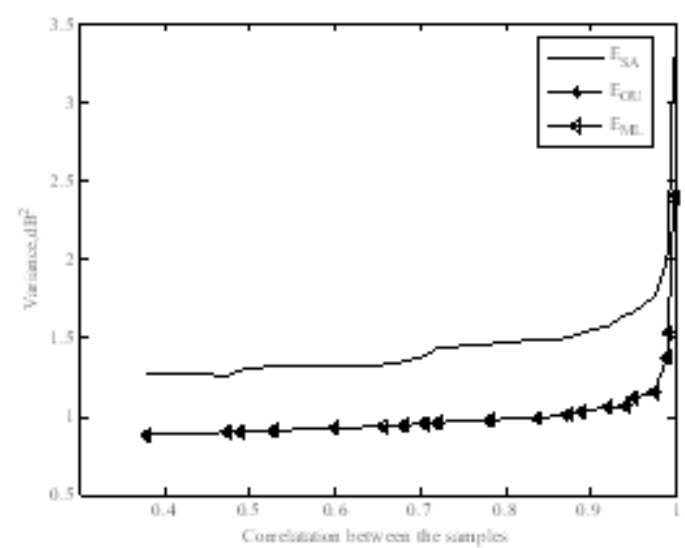

Fig. 10. Variance of the three estimators $\left[f_{m}=15 \mathrm{~Hz}, 30 \mathrm{~Hz}\right.$ $.300 \mathrm{~Hz}$, Number of samples per second $N_{f}=1 / T_{p}=1500$, $N=20, M=20]$ 